\title{
The Mechanical Behavior of Polyline Crease Origami Self-Locking Structure under Quasi-Static Compressive Loading
}

\author{
Peiwen Zhang $\mathbb{D},{ }^{1}$ YuLong He $\mathbb{D}^{2},{ }^{2}$ Huawei Yang $\mathbb{D}^{1},{ }^{1}$ and Xuxian Yan $\mathbb{D}^{1}$ \\ ${ }^{1}$ College of Management Science and Engineering, Shanxi University of Finance and Economics, Taiyuan 030006, China \\ ${ }^{2}$ Institute of Applied Mechanics, College of Mechanical and Vehicle Engineering, Taiyuan University of Technology, \\ Taiyuan 030024, China \\ Correspondence should be addressed to Peiwen Zhang; zhangpwphd@163.com
}

Received 10 November 2021; Accepted 17 January 2022; Published 18 February 2022

Academic Editor: Ivan Giorgio

Copyright (c) 2022 Peiwen Zhang et al. This is an open access article distributed under the Creative Commons Attribution License, which permits unrestricted use, distribution, and reproduction in any medium, provided the original work is properly cited.

\begin{abstract}
The unique mechanical behavior of polyline crease (PLC) origami structures inspired by the Tachi-Miura polyhedron (TMP) is investigated. We explore the potential usage as mechanical metamaterials that exhibit negative Poisson's ratio, self-locking mechanism, and load capacity simultaneously. Poisson's ratio, locked folding ratio, dimensionless force and folding ratio relationship, and elastic-plastic response during its compressive process (rigid folding motion or plastic bending/buckling) are investigated analytically and experimentally, respectively. The effect of original folding angle and the length-to-height ratio on negative Poisson's ratio, locked folding ratio, dimensionless force, deformation modes, and the mean compression force are also considered. Based on the experiments, an approximate theoretical study has also been conducted, which shows a reasonable correlation with the experimental results. The results are significant to guide the engineering applications of PLC self-locking origami structures.
\end{abstract}

\section{Introduction}

Origami-inspired metamaterial has become more and more important in the manmade material field due to their macroscale mechanical properties, and this is primarily determined by designing microscale structures purposefully [1-7]. In recent years, straight crease origami structures have been investigated extensively due to the feature of lightweight, high compact ratio, and ease to fold and deploy. Different from straight crease (SC) origami, curve crease pattern (CC) origami can be designed to be curved shape to occupy specific space [8-10], which makes them desirable for engineering applications. Recently, origami has been identified as a platform to implement programmable mechanical metamaterials. Origami has received significant interest in diverse applications because of its excellent properties that have been verified, including tunable Poisson's ratio and stiffness $[2,11-15]$, programmable collapse
[5], self-locking [16-18], and multi-stability [19]. A metamaterial is generated by stacking of individual units, in which preserving the folding kinematics between the sheets was described by Schenk [11]. Because of the special geometries, the origami metamaterials usually exhibit a negative Poisson ratio under in-plane deformations [9]. There are many materials or structures that also have negative Poisson's ratio properties, such as kirigami $[20,21]$, origami [22], and other materials [23-25]. Some material properties can be enhanced as a result of having a negative Poisson ratio, for example, the indentation resistance or hardness [23]. Poisson's ratio switching is another important property that has been found recently for the Morph pattern, in which its Poisson's ratio can change from negative infinity to positive infinity theoretically [26]. The quasi-static and dynamic mechanical behaviors at large plastic strains of the metal-based cellular materials have been widely studied to bring insight into their energy absorption capacity [27-35]. 
Moreover, previous investigations demonstrated that the energy absorption capacity depends on the material topology [36]. The relationship between the geometrical configurations of the origami metamaterials and overall mechanical properties has been also examined [37-41].

Polyline crease (PLC) origami differs from SC origami and CC origami, in that the folded surface of the pattern keeps rigid motion before it reaches locked strain. Then, the folded surface is bent during the folding process after it reached the locked folding angle (or locked strain). Limited studies on the mechanical performance of such geometries have been conducted, in part because of the difficulty in parameterizing and modeling the pattern geometry. This study presents a new nondevelopable degree 4 unit cell. It is developed with a two-stage method that converts straight crease Miura-derivative base geometries into polyline crease geometries. The first stage involves fitting a polyline to a prismatic origami surface, and the second stage involves subdividing this curve into prismatic rigid origami slices with an enforced common configuration angle at slice interfaces. A generic degree 4 vertex origami possesses one continuous degree of freedom for rigid folding, and this folding process can be stopped when two of its facets bind together. Such facet binding will induce self-locking so that the overall structure stays at a prespecified configuration without additional locking elements or actuators. Selflocking offers many promising properties, such as programmable deformation ranges and piecewise stiffness jumps, which could significantly advance many adaptive structural systems. However, despite its excellent potential, the origami self-locking features have not been well studied, understood, and used.

In this study, one new PLC origami unit cell with 4 interlayers (PLC-IL4) was designed firstly and then Poisson's ratio was investigated. By exploiting various geometrical configurations, the self-locking mechanism was investigated, which can effectively induce piecewise stiffness jumps. The polyline crease origami offers the origami structures with extraordinary attributes that the straight crease origami does not have, such as the programmable locked folding ratios. The specimens with different original folding angles of the designed PLC-IL4 origami unit cell are then manufactured using cardboard. The effects of original folding angle of PLCIL4 origami structure on the quasi-static behavior were investigated experimentally. Finally, the experimental results of PLC-IL4 origami structure were compared with that of theoretical prediction. The results of this research provide new scientific knowledge for the design and utilization of PLC origami structures for many potential engineering applications.

\section{Negative Poisson's Ratio}

To calculate $W, B$, and $H$ under various folding configurations, a half model of the PLC-IL4 origami structure was considered, as shown in Figure 1(e), which corresponds to Figures 1(a)-1(d). The folding angles $\gamma$ and $\beta$ are functions of $\alpha$ (original folding angle) and $\theta$ (varies by the degree of folding). Based on the geometry described in Figure $1, W, B$, and $H$ are obtained as follows:

$$
\begin{aligned}
W & =l+l \cdot \cos \theta+\frac{D / 4}{\tan \alpha_{1}}+\frac{D / 4}{\tan \alpha_{2}}, \\
B & =l \sin \theta+\frac{D}{4} \sin \beta_{1}+\frac{D}{4} \sin \beta_{2}, \\
\frac{H}{2} & =\frac{D}{4} \cos \beta_{1}+\frac{D}{4} \cos \beta_{2}, \\
\tan \frac{\theta}{2} & =\tan \alpha_{1} \cdot \sin \beta_{1} \\
& =\tan \alpha_{2} \cdot \sin \beta_{2}, \\
\sin \frac{\theta}{2} & =\sin \alpha_{1} \cdot \cos \frac{\gamma_{1}}{2} \\
& =\sin \alpha_{2} \cdot \cos \frac{\gamma_{2}}{2} .
\end{aligned}
$$

Poisson's ratios of the PLC-IL4 are investigated by defining them as follows:

$$
\begin{aligned}
& v_{Z X}=-\frac{(d W / W)}{d H / H}, \\
& v_{Z Y}=-\frac{(d B / B)}{d H / H} .
\end{aligned}
$$

Differentiating equation (1) with respect to the folding angles and plugging them into equation (2), Poisson's ratios were obtained as follows:

$$
\begin{aligned}
& v_{Z X}=-\frac{2 l \sin \theta \tan \alpha_{1} \cos \beta_{1} \cos ^{2} \theta / 2\left(\cos \beta_{1}+\cos \beta_{2}\right)}{(l+l \cos \theta+D / 4 \tan \alpha 1+D / 4 \tan \alpha 2)\left(\sin \beta_{1}+\sin \beta_{2} \tan \alpha_{1} \cos \beta_{1} / \tan \alpha_{2} \cos \beta_{2}\right)}, \\
& v_{Z Y}=\frac{\left(8 l \cos \theta \tan \alpha_{1} \cos \beta_{1} \cos ^{2} \theta / 2+D \cos \beta_{1}+D \tan \alpha_{1} / \tan \alpha_{2} \cos \beta_{1}\right)\left(\cos \beta_{1}+\cos \beta_{2}\right)}{\left(4 l \sin \theta+D \sin \beta_{1}+D \sin \beta_{2}\right)\left(\sin \beta_{1}+\sin \beta_{2} \tan \alpha_{1} \cos \beta_{1} / \tan \alpha_{2} \cos \beta_{2}\right)} .
\end{aligned}
$$

2.1. The Effect of Original Folding Angle on Poisson's Ratio. Poisson's ratio of Miura-ori has been verified to be negative as testified by some theoretical studies $[11,12,19,26]$. A similar conclusion was obtained in this study, and the PLCIL4 origami unit cells with different original folding angles exhibit negative Poisson's ratio in the folding process. The 


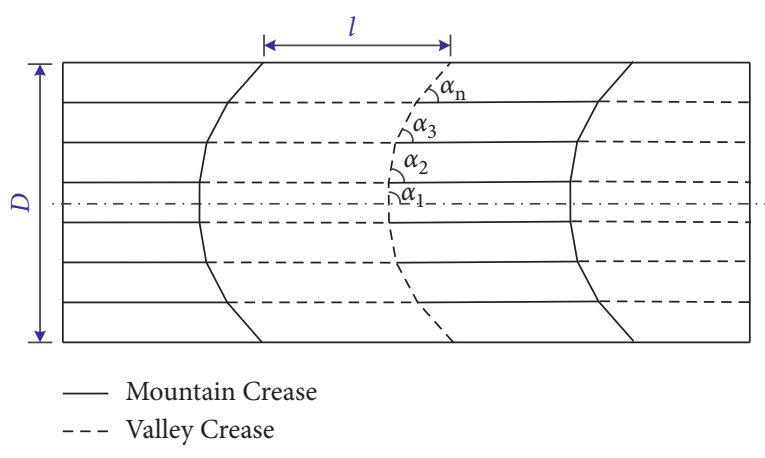

(a)
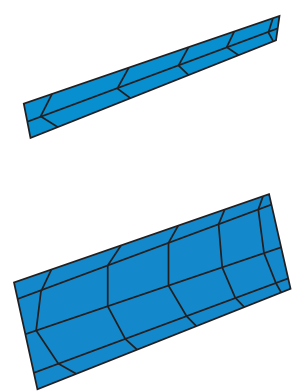

— Mountain Crease

- - - Valley Crease

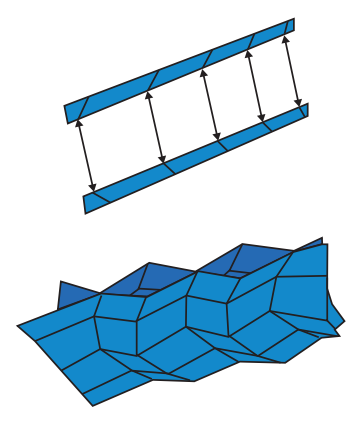

(c)

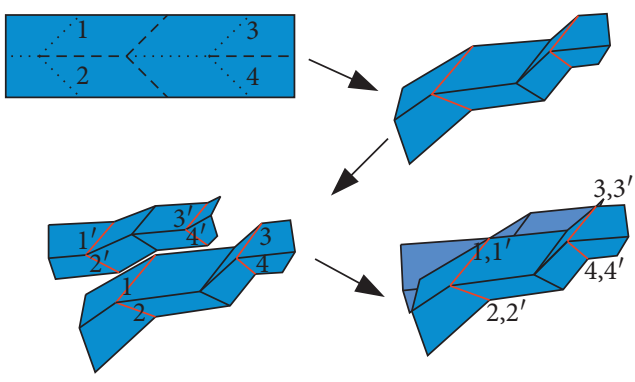

- Mountain Crease - - - Valley Crease

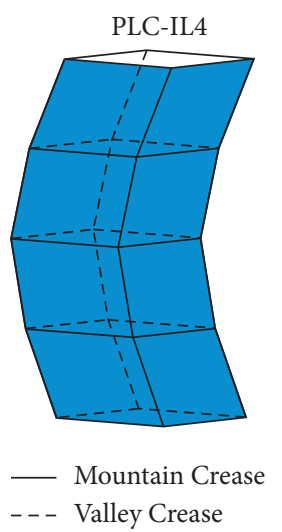

(d)

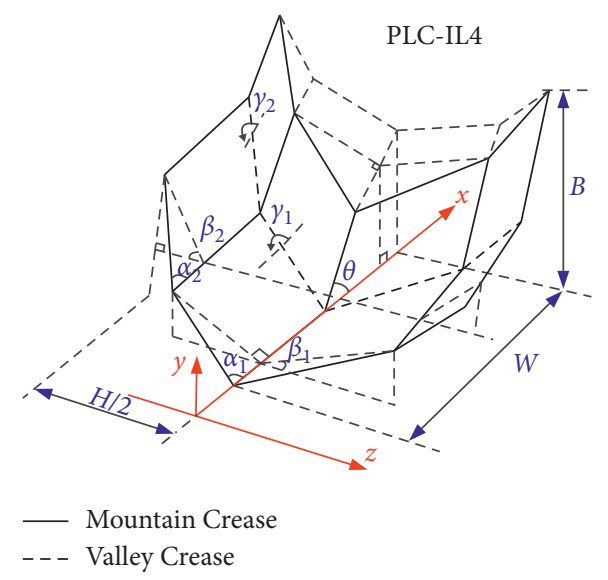

(e)

Figure 1: Illustrations of PLC-IL4 origami structure. (a) The PLC origami pattern in its planar state. (b) A Miura-ori in its folded state. (c) The fabrication process of a PLC unit cell. (d) A unit cell of a PLC origami fabricated from two thin cardboards bonded together. (e) The sketch of a half model of PLC origami unit cell. The dimension of the unit cell is $w, b$, and $h$ in $x$-, $y$-, and $z$-directions, respectively.

effect of original folding angle on Poisson's ratio was investigated using 9 different angle values such as $\alpha_{1}=50^{\circ}$, $55^{\circ}, 60^{\circ}, 65^{\circ}, 70^{\circ}, 75^{\circ}, 80^{\circ}$, and $85^{\circ}$ and $\alpha_{2}=\alpha_{1}-5^{\circ}$, while the length and width were fixed as $l=10 \mathrm{~mm}$ and $D=40 \mathrm{~mm}$. In Figure 2, the range of negative Poisson's ratio is shown, and it can be seen that the value of $v_{\mathrm{ZX}}$ at the start of folding stage increases, but the value of $v_{\mathrm{ZY}}$ decreases with the decreasing original folding angle. The region of negative Poisson's ratio is also decreasing with the decreasing original folding angle. The main reason for this phenomenon is that the reentrant extent of the PLC-IL4 origami structure with the small original folding angle $\alpha_{1}$ is more obvious than that with big angle values. So, the lateral displacement of origami cell with the small original folding angle is larger. It is noteworthy that the color range of Poisson's ratio $\left(v_{\mathrm{ZX}}\right.$ and $\left.v_{\mathrm{ZY}}\right)$ occupied will decrease as the original folding angle $\alpha_{1}$ increases, which corresponded to the decreasing folding ratio. The main reason is that this PLC origami unit cell should satisfy the geometrical relations as shown in equation (1). 

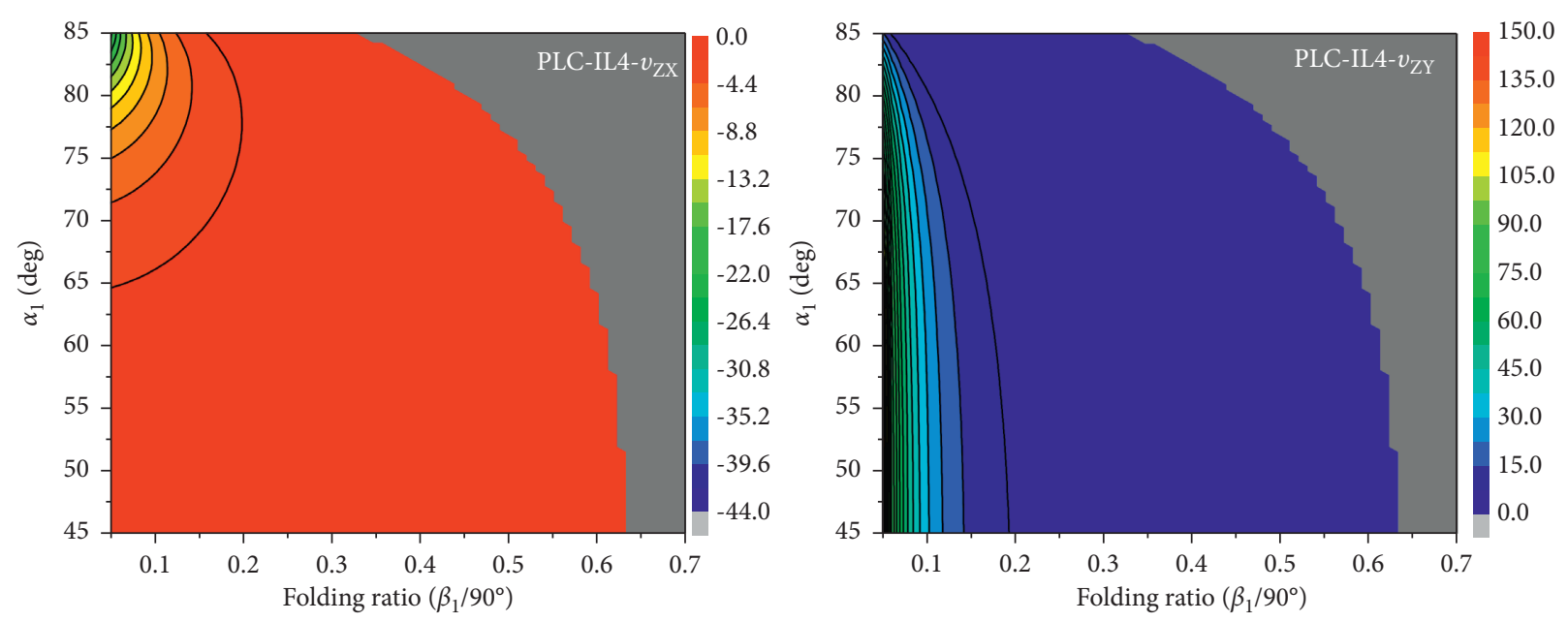

FIGURe 2: Contour plot of out-plane Poisson's ratio $\left(v_{\mathrm{ZX}}, v_{\mathrm{ZY}}\right)$ of PLC-IL4 origami unit cell with different original folding angles.

$$
\begin{aligned}
\tan \frac{\theta}{2} & =\tan \alpha_{1} \cdot \sin \beta_{1} . \\
& =\tan \alpha_{2} \cdot \sin \beta_{2}
\end{aligned}
$$

Thus,

$$
\sin \beta_{2}=\frac{\tan \alpha_{1}}{\tan \alpha_{2}} \cdot \sin \beta_{1} .
$$

It should be noted that while $\beta_{2} \in\left[0 ; 90^{\circ}\right]$, the range of $\beta_{1}$ is limited to satisfy $0 \leq \beta_{1} \leq \operatorname{arcsintan} \alpha_{2} / \tan \alpha_{1}$. This is to avoid the collision between two adjacent planes during the folding process. For example, while $\alpha_{1}=85^{\circ}$ and $\alpha_{2}=\alpha_{1}-5^{\circ}=80^{\circ}$, the range of $\sin \beta_{1}$ is $0 \leq \sin \beta_{1} \leq 0.4962$, which corresponded to $0 \leq \beta_{1} \leq 29.75^{\circ}$. Then, the range of folding ratio $\left(\beta_{1} / 90^{\circ}\right)$ is $0 \leq \beta_{1} / 90^{\circ} \leq 0.331$.

A schematic of a special folding rate is shown in Figure 3. The folding rate is defined as the ratio of the angle $\beta_{1}$ to $90^{\circ}$. For a specimen, while its $\beta_{1}=45^{\circ}$ in the folding process, the corresponding folding rate is 0.5 .

The analytical contour plot of locked folding ratio as a function of continuous $\alpha_{1}-\alpha_{2}$ and the original folding angle $\alpha_{1}$ is shown in Figure 4. The analytical contour plot provides a quantitative tool for designing on request self-locking behavior and original folding angle, which can be used to optimize PLC-IL4 origami programmable structures. It is seen that the locked folding ratio decreases with the increasing original folding angle $\alpha_{1}$ while $\alpha_{1}-\alpha_{2}$ is fixed. At a certain locked folding ratio, the PLC-IL4 origami structure can be designed to different geometrical configurations. For example, while the engineering requirement of PLC-IL4 origami structure locked folding ratio is 0.4 , we only need to draw a parallel line of $x$-axis that through point 0.4 . Then, different geometrical configurations could be obtained (such as $\alpha_{1}=74^{\circ}, \alpha_{2}=64^{\circ} ; \alpha_{1}=81.1^{\circ}$ and $\alpha_{2}=75.1^{\circ}$ ). Similarly, if the original folding angle is fixed as $\alpha_{1}=70^{\circ}$, we also can obtain different locked folding ratios by changing the value of $\alpha_{1}-\alpha_{2}$. It also can be found that the locked folding ratio will be equal to zero (which means the self-locking behavior eventually disappears), while the original folding angle $\alpha_{1}$

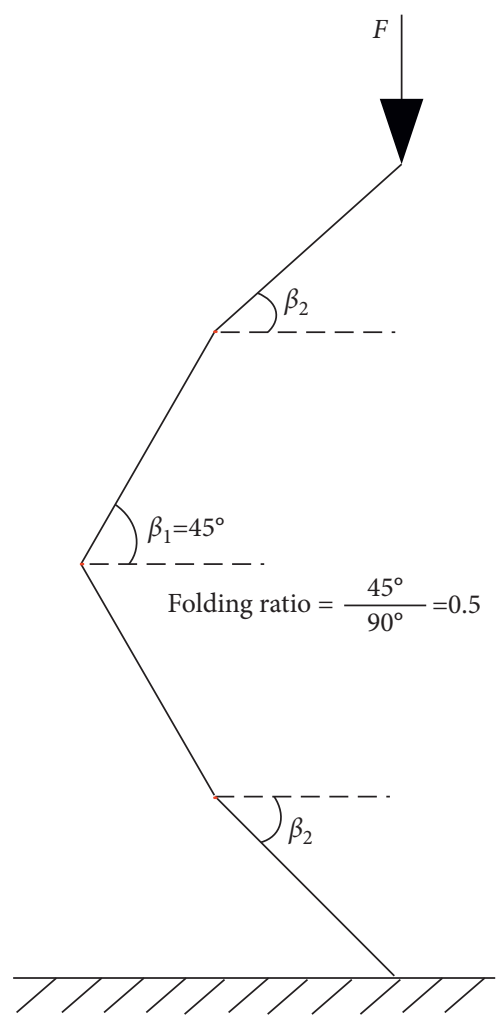

Figure 3: A schematic example of a specific folding ratio.

increases to $90^{\circ}$. This phenomenon indicates that the PLCIL4 origami structure will degenerate to another new type of PLC structure in which the self-locked behavior does not exist, which can be exploited for the purpose of intelligent control and metamaterial design.

2.2. The Effect of Length-to-Width Ratio on Poisson's Ratio. The length-to-width ratio also can exert an influence on Poisson's ratio of PLC origami unit cell, which has been reported in the investigation of Miura-ori [12]. By exploiting different length-to-width $(l / D)$ ratios, the effect of it on 


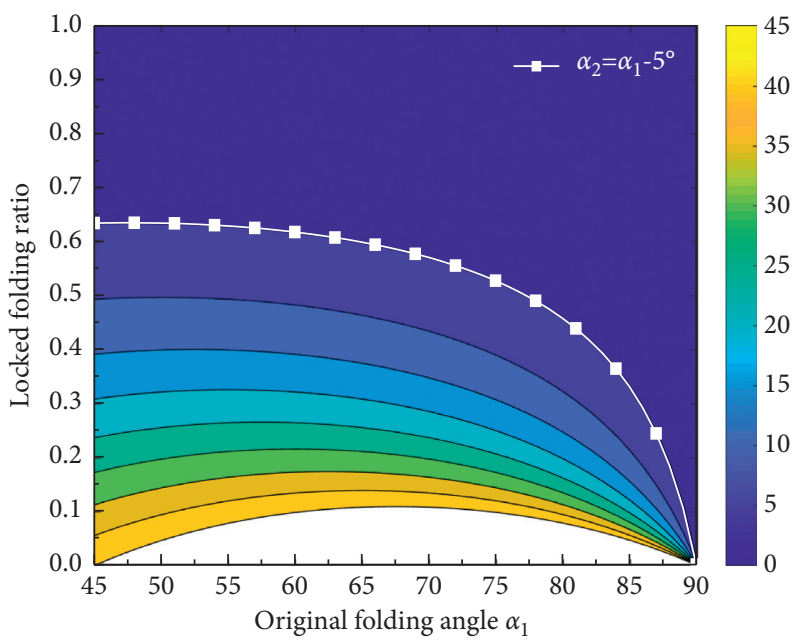

Figure 4: Locked folding ratio change in PLC-IL4 origami structure with different $\alpha_{1}-\alpha_{2}$ values.

Poisson's ratio was investigated, as shown in Figure 5. Five unit cell widths (10 mm, $20 \mathrm{~mm}, 30 \mathrm{~mm}, 40 \mathrm{~mm}$, and $50 \mathrm{~mm}$ ) were used, while the length and the original folding angle were fixed as $l=10 \mathrm{~mm}$, and $\alpha_{1}=60^{\circ}$ and $\alpha_{2}=55^{\circ}$. Then, five different length-to-width $(l / D)$ ratios were obtained through calculation. The folding ratio of this PLC-IL4 origami structure only reaches 0.617 due to the self-locking behavior. It is observed that the absolute values of Poisson's ratio $v_{\mathrm{ZX}}$ increase with the increasing $l / D$ ratio, but the area that the negative Poisson's ratio occupied is decreasing. It means that the $v_{\mathrm{ZX}}$ of PLC-IL4 origami unit cell with different length-towidth ratios will decrease to remain close to zero when the folding ratio increases to 0.4 . The $v_{\mathrm{ZY}}$ of PLC-IL4 origami unit cell with different length-to-width ratios is tending to zero early and does not exhibit negative values in the folding process. This phenomenon suggests that the length-to-width ratio has less impact on $v_{\mathrm{ZY}}$ than that on $v_{\mathrm{ZX}}$.

\section{The Dimensionless Force and Folding Ratio Relationship}

The force and folding ratio relationship was investigated to validate the effect of original folding angle on the PLC-IL4 origami unit cell, as shown in Figure 6. The PLC-IL4 origami unit cell calculation model was established by rigid plates connected by torsional spring along the crease lines. The folding behavior of the PLC-IL4 origami unit cell was considered under a uniaxial force $(F)$ in the $z$-direction. By applying virtual displacement $(\delta u)$ to the PLC-IL4 unit cell and using the principle of virtual work, the following equation was obtained.

$$
F \delta u=2 n_{h_{1}} M_{h_{1}} \delta \beta_{1}+n_{h_{2}} M_{h_{2}} \delta \beta_{2}+n_{b_{1}} M_{b_{1}} \delta \gamma_{1}+n_{b_{2}} M_{b_{2}} \delta \gamma_{2} .
$$

The bending moments $M_{\mathrm{h}}$ and $M_{\mathrm{b}}$ are along horizontal and inclined crease lines, respectively, and $n_{h_{1}}, n_{h_{2}}, n_{b_{1}}, n_{b_{2}}$ are the number of the horizontal and inclined crease lines corresponded to $\beta_{1}, \beta_{2}, \gamma_{1}$, and $\gamma_{2}$; the relationship between bending moment and angle is assumed as linear:

$$
\begin{aligned}
& M_{h}=k_{\theta}\left(\beta-\beta_{0}\right) ; \\
& M_{b}=k_{\theta}\left(\gamma-\gamma_{0}\right),
\end{aligned}
$$

where $k_{\theta}$ is a spring constant, and $\beta$ and $\gamma$ are the original folding angles for horizontal and inclined crease lines, respectively.

By applying variation to equation (1) with respect to the folding angles, the following relationships can be obtained:

$$
\begin{aligned}
\delta \theta & =2 \tan \alpha_{1} \cos ^{2} \frac{\theta}{2} \cos \beta_{1} \delta \beta_{1} \\
& =2 \tan \alpha_{2} \cos ^{2} \frac{\theta}{2} \cos \beta_{2} \delta \beta_{2}, \\
\frac{1}{2} \cos \frac{\theta}{2} \delta \theta & =-\frac{1}{2} \sin \alpha_{1} \sin \frac{\gamma_{1}}{2} \delta \gamma_{1} \\
& =-\frac{1}{2} \sin \alpha_{2} \sin \frac{\gamma_{2}}{2} \delta \gamma_{2} .
\end{aligned}
$$

Therefore,

$$
\begin{aligned}
& \delta \beta_{2}=\frac{\tan \alpha_{1} \cos \beta_{1}}{\tan \alpha_{2} \cos \beta_{2}} \delta \beta_{1}, \\
& \delta \gamma_{1}=-\frac{2 \cos ^{3} \theta / 2 \cos \beta_{1}}{\cos \alpha_{1} \sin \gamma_{1} / 2} \delta \beta_{1}, \\
& \delta \gamma_{2}=-\frac{2 \cos ^{3} \theta / 2 \tan \alpha_{1} \cos \beta_{1}}{\sin \alpha_{2} \sin \gamma_{2} / 2} \delta \beta_{1} .
\end{aligned}
$$

The height of the PLC-IL4 is as follows:

$$
H_{0}-u=\frac{D}{2} \cos \beta_{1}+\frac{D}{2} \cos \beta_{2} .
$$

Then,

$$
\delta u=\frac{1}{2} D \sin \beta_{1} \delta \beta_{1}+\frac{1}{2} D \sin \beta_{2} \delta \beta_{2} .
$$



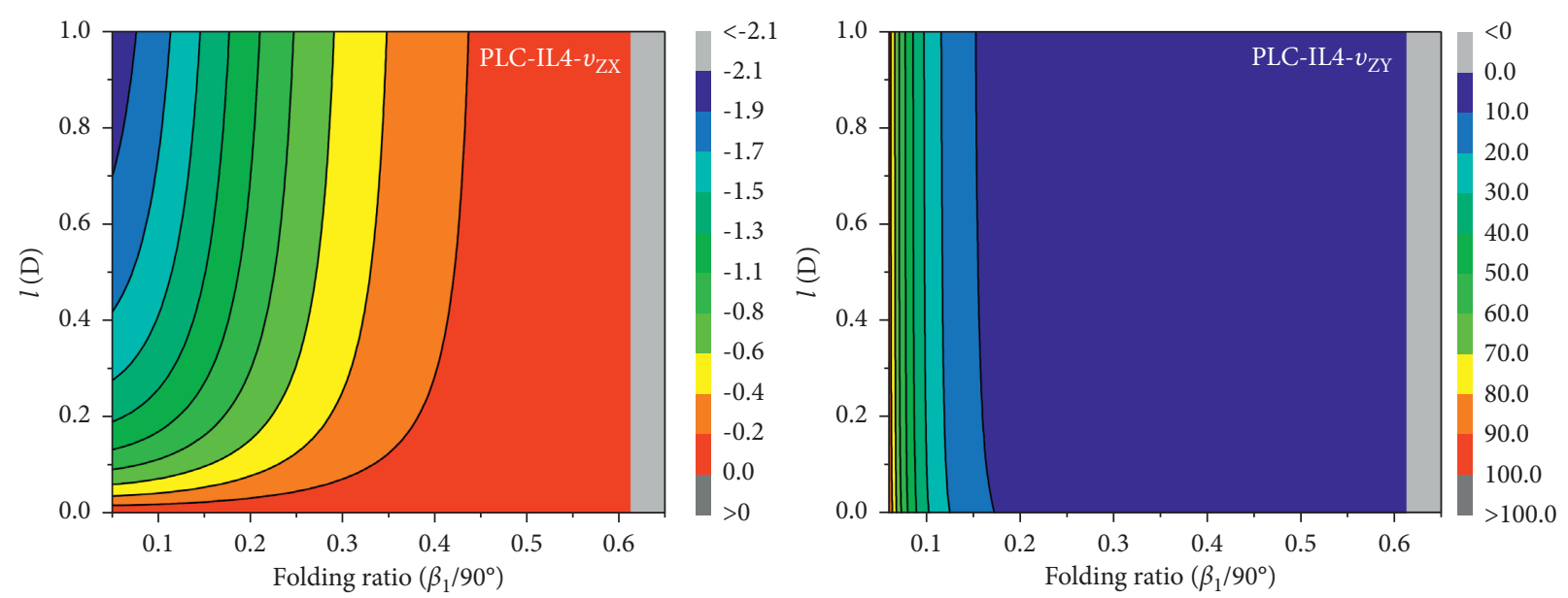

FIgURe 5: Contour plot of out-plane Poisson's ratio $\left(v_{\mathrm{ZX}}, v_{\mathrm{ZY}}\right)$ of PLC origami unit cell with different $l / D$ ratios.
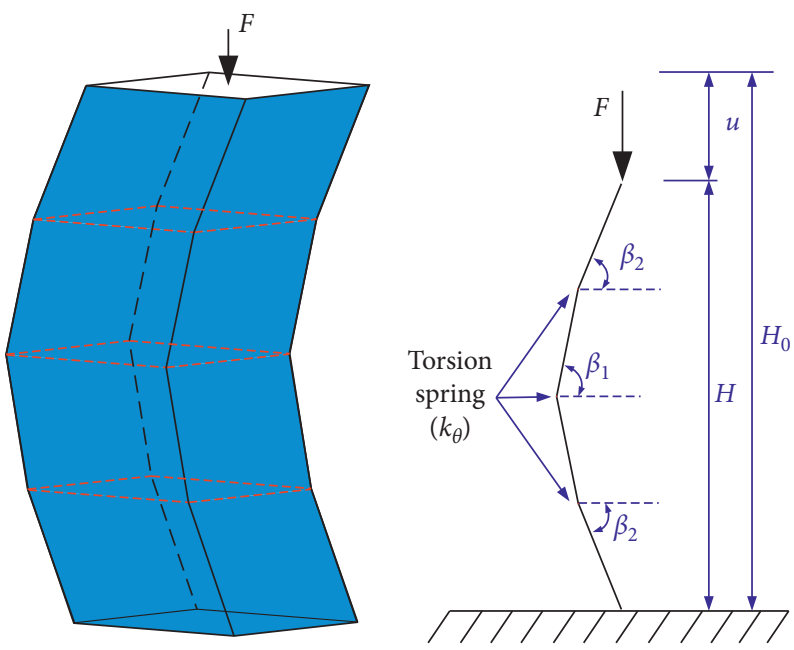

FIGURE 6: Sketch of PLC origami unit cell and calculation model with a torsional spring.

Substituting equations (7)-(12) into equation (6), the relationship between force and displacement of PLC-IL4 can be obtained as follows:

$$
\begin{aligned}
\frac{F}{\left(k_{\theta} / D\right)}= & \frac{2}{\left(\sin \beta_{1}+\tan \alpha_{1} \cos \beta_{1} / \tan \alpha_{2} \cos \beta_{2} \sin \beta_{2}\right)} \\
& \left\{\begin{array}{l}
2 n_{h_{1}}\left[\left(\beta_{1}-\beta_{1}^{0}\right)\right]+n_{h_{2}}\left[\left(\beta_{2}-\beta_{2}^{0}\right)\right] \frac{\tan \alpha_{1} \cos \beta_{1}}{\tan \alpha_{2} \cos \beta_{2}} \\
-n_{b_{1}}\left[\left(\gamma_{1}-\gamma_{1}^{0}\right)\right]\left(\frac{2 \cos ^{3} \theta / 2 \cos \beta_{1}}{\cos \alpha_{1} \sin \gamma_{1} / 2}\right)-n_{b_{2}}\left[\left(\gamma_{2}-\gamma_{2}^{0}\right)\right]\left(\frac{2 \cos ^{3} \theta / 2 \tan \alpha_{1} \cos \beta_{1}}{\sin \alpha_{2} \sin \gamma_{2} / 2}\right)
\end{array}\right\} .
\end{aligned}
$$


The numbers of the torsional spring along horizontal and inclined crease lines are $n_{h_{1}}=4, n_{h_{2}}=8, n_{b_{1}}=8$, and $n_{b_{2}}=8$, respectively. Then, the relationship between force and displacement of PLC-IL4 can be obtained finally as follows:

$$
\begin{gathered}
\frac{F}{\left(k_{\theta} / D\right)}=\frac{16}{\left(\sin \beta_{1}+\tan \alpha_{1} \cos \beta_{1} / \tan \alpha_{2} \cos \beta_{2} \sin \beta_{2}\right)} \\
\left\{\begin{array}{c}
{\left[\left(\beta_{1}-\beta_{1}^{0}\right)\right]+\left[\left(\beta_{2}-\beta_{2}^{0}\right)\right] \frac{\tan \alpha_{1} \cos \beta_{1}}{\tan \alpha_{2} \cos \beta_{2}}-\left[\left(\gamma_{1}-\gamma_{1}^{0}\right)\right]\left(\frac{2 \cos ^{3} \theta / 2 \cos \beta_{1}}{\cos \alpha_{1} \sin \gamma_{1} / 2}\right)} \\
-\left[\left(\gamma_{2}-\gamma_{2}^{0}\right)\right]\left(\frac{2 \cos ^{3} \theta / 2 \tan \alpha_{1} \cos \beta_{1}}{\sin \alpha_{2} \sin \gamma_{2} / 2}\right)
\end{array}\right\} .
\end{gathered}
$$

The force and folding ratio relationships of PLC-IL4 origami unit cell with four different original folding angles $\left(\alpha_{1}=55^{\circ}, 65^{\circ}, 75^{\circ}\right.$, and $85^{\circ}$ and $\alpha_{2}=\alpha_{1}-5^{\circ}$ ) based on equation (12) are presented in Figure 7. It is observed that the relationship between the normalized force and the folding ratio is not unique. The normalized force of PLC-IL4 unit cell with $\alpha_{1}=55^{\circ}$ and $65^{\circ}$ increases gradually, while that of PLC-IL4 unit cell with $\alpha_{1}=75^{\circ}$ and $85^{\circ}$ tends to develop a higher peak load before the load drops during the folding process. This indicated that the PLC-IL4 unit cell with $\alpha_{1}=75^{\circ}$ and $85^{\circ}$ exhibits local structural softening, which is called "type II" structures, while the PLC-IL4 unit cell with $\alpha_{1}=55^{\circ}$ and $65^{\circ}$ does not exhibit local structural softening, which is called "type I" structures [42]. There is a local minimum point of normalized force corresponding to the folding ratio for the PLC-IL4 unit cell with $\alpha_{1}=75^{\circ}$ and $85^{\circ}$, while the normalized force increases in both directions. This indicates that the PLC-IL4 origami unit cell under $\alpha_{1}=75^{\circ}$ and $\alpha_{1}=85^{\circ}$ also exhibits two stable configurations, which are consistent with the results in [19].

\section{Experimental Investigation}

4.1. The Mechanical Properties of Cardboard. All the specimens were cut from the cardboard KZ-230G with $0.3 \mathrm{~mm}$ thickness. The density of the cardboard is $0.8016 \mathrm{~g} / \mathrm{cm}^{3}$. Quasi-static tensile tests at $20 \mathrm{~mm} / \mathrm{min}$ were conducted to determine the properties of the cardboard using an Instron testing system (Instron 5544, Instron, Norwood, MA, USA). The dimensions of samples are shown in Figure 8(a). Force and displacement were recorded by the Instron testing system. Figure 8(b) shows the force-displacement curves of the specimens. It can be found that the test results are very close to each other. Therefore, the average mechanical properties of all specimens are as follows: $E$ (Young's modulus $)=4.02 \mathrm{GPa} ; \sigma_{\mathrm{y}}$ (yield stress $)=12.81 \mathrm{MPa} ; \sigma_{\mathrm{u}}(\mathrm{ul}-$ timate stress $)=34.19 \mathrm{MPa}$; and $n$ (hardening exponent $)=$ 0.5017 .

4.2. Specimen Preparation. PLC-IL4 origami unit cell specimens, $60 \mathrm{~mm}$ long, with a width also of $60 \mathrm{~mm}$, were fabricated from two thin cardboards bonded together. A photograph of samples with an original folding angle $\left(65^{\circ}\right)$ is shown in Figure 9. A total of 40 samples were fabricated. To examine the effect of original folding angle on the forcedisplacement relationship, five nominally identical specimens were used for each test condition. Samples were identified by a unique label; e.g., PLC-IL4-50-1 indicates a sample with polyline crease pattern, divides height into 4 equal parts, an original folding angle $\alpha_{1}=50^{\circ}$, and is the first specimen used to investigate the effect of folding angle value.

4.3. Test Result Discussion. To gain insight into the effect of original folding angle, the PLC-IL4 samples of eight different angle values were tested at quasi-static compression of $20 \mathrm{~mm} / \mathrm{min}$, as summarized in Figure 10(a). It is seen that the force-displacement curve exhibits initial peak, while the original folding angle increases over $65^{\circ}$. The load capacity of the samples decreases with the increasing original folding angle, while their displacement exceeds the rigid folding part. The possible reason is that the PLC-IL4 unit cell with small $\alpha_{1}$ exhibits local structural softening, while that with larger does not exist. Figure 10(b) presents the deformation process of the sample with an original folding angle $\left(\alpha_{1}=70^{\circ}\right.$, $\alpha_{2}=65^{\circ}$ ). The compressive force of the sample is less than $1 \mathrm{~N}$ until the displacement increases to $32 \mathrm{~mm}$ (corresponded to locked folding ratio of 0.615 ), which is mainly caused by the friction in the rigid folding stage, but the force increases sharply after the displacement exceeds the critical value of rigid folding motion due to the plastic deformation.

The experimental locked folding ratio of specimens with different original folding angles and the theoretical prediction values is plotted in Figure 11. As mentioned above, the locked folding ratio decreases with the increasing original folding angle $\alpha_{1}$, while $\alpha_{1}-\alpha_{2}$ is fixed. As shown in Figure 11, it is clear that all the experimental data points are concentrated basically around the theoretical prediction line, herein indicating that the experimental measurements can fit theoretical predictions well.

Figure 12 shows the typical deformation modes of PLCIL4 origami structure with different original folding angles. It can be seen that the original folding angle value has less influence on the deformation modes. The forms of plastic hinge only occur in the adjacent inclined creases, while the 


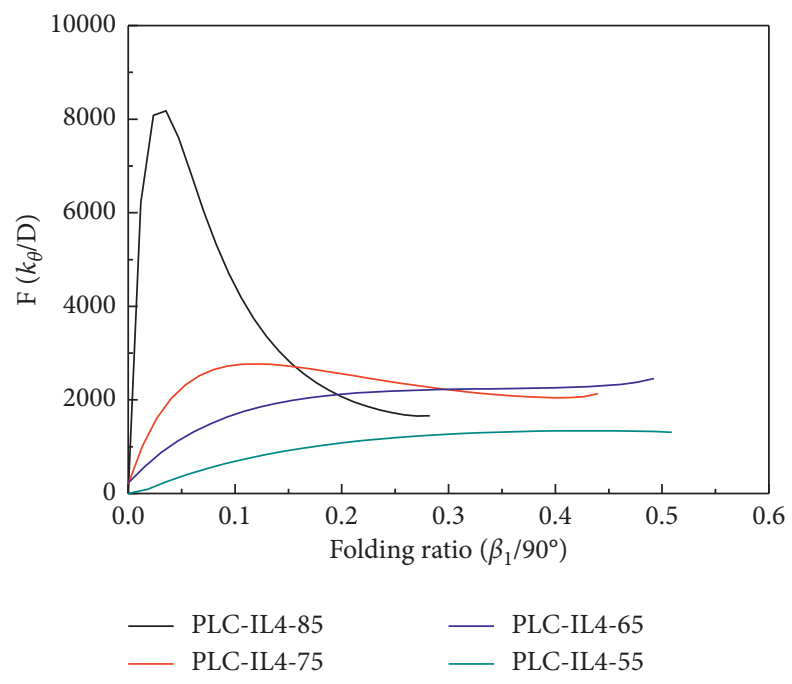

FIgURE 7: Effect of original folding angle on normalized force and folding ratio relationship.
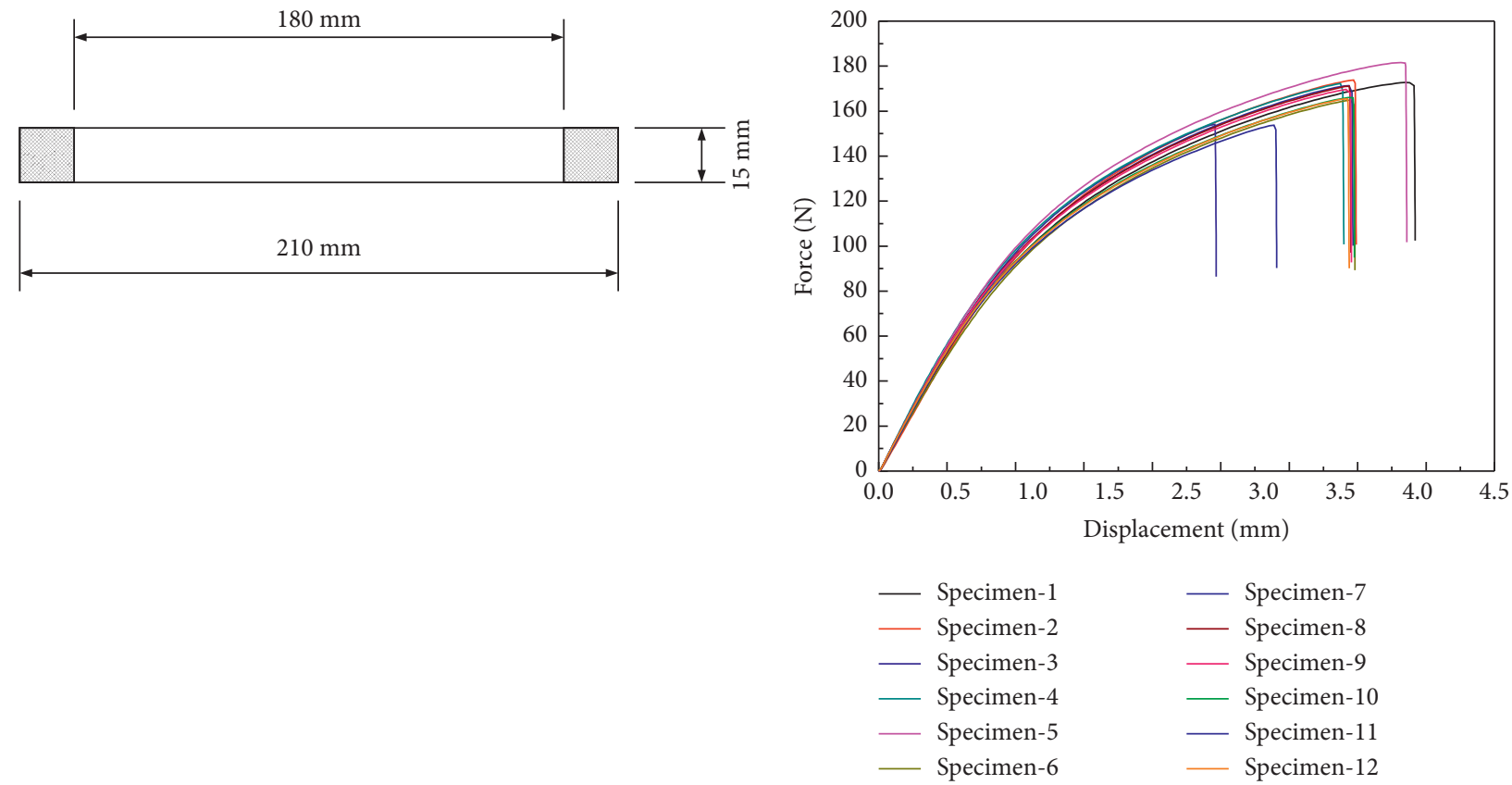

(a)

(b)

Figure 8: Quasi-static testing of cardboard KZ-230G: (a) sketch of sample dimensions; (b) force-displacement curves obtained from the tests of samples.

sample compressive process exceeds the rigid folding motion (which corresponded to folding ratio that exceeds the locked folding ratio). The main reason is that the value of $\alpha_{1}-\alpha_{2}$ is relatively small, which will result in a little plastic area. Thus, the deformation modes of all the samples are small elasticplastic deformations that are very similar to each other.

\section{Theoretical Analysis}

The typical deformation mode for theoretical prediction of PLC-IL4 structure with different original folding angles is proposed based on experiments, as shown in Figure 13. Then, a simple mathematical formula was established to 


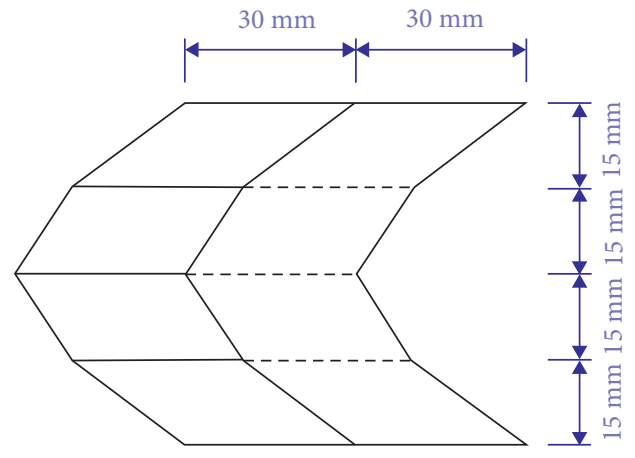

(a)

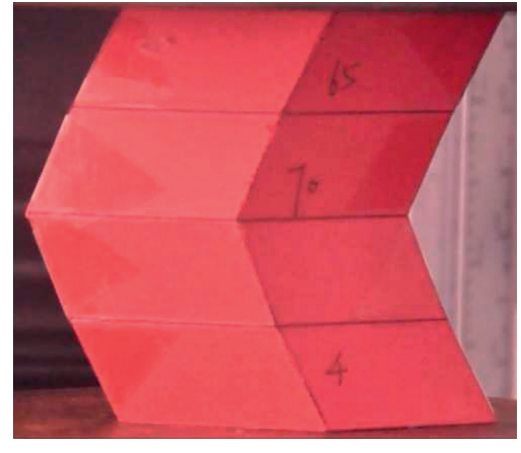

(b)

Figure 9: PLC-IL4 samples: (a) sketch of sample dimensions; (b) a sample of PLC-IL4-70-4 with an original folding angles $\alpha_{1}=70^{\circ}$ and $\alpha_{2}=65^{\circ}$.

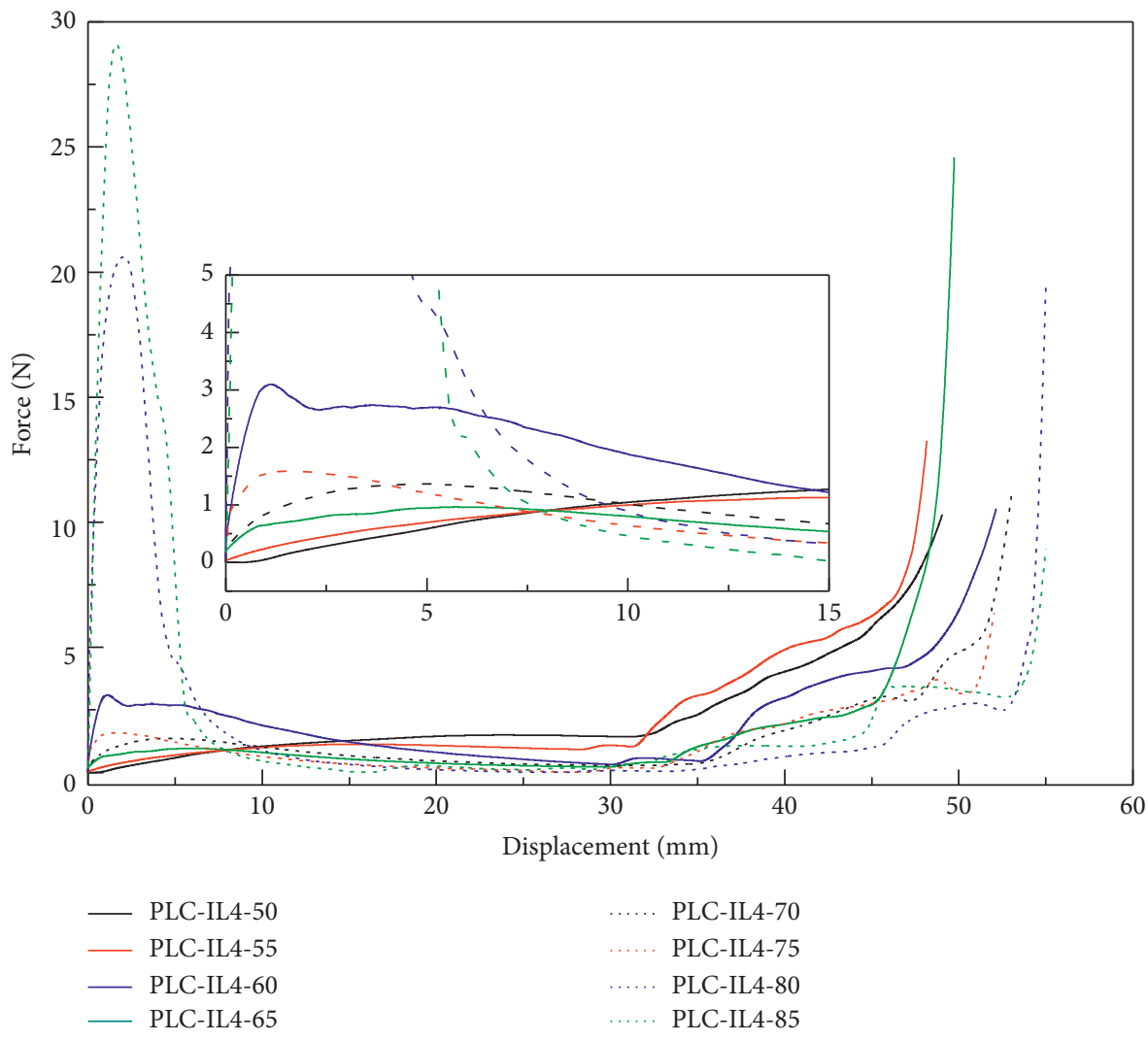

(a)

Figure 10: Continued. 


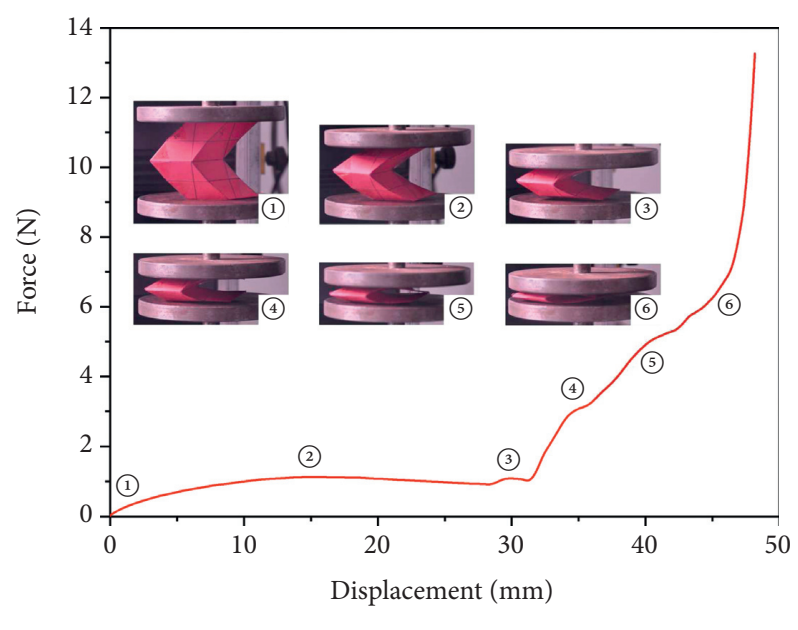

PLC-IL4-55

(b)

FiguRE 10: Typical force-displacement history curves: (a) quasi-static compressive response of samples with five different original folding angles; (b) deformation process of the sample with an original folding angle $\left(\alpha_{1}=60^{\circ}, \alpha_{2}=55^{\circ}\right)$.

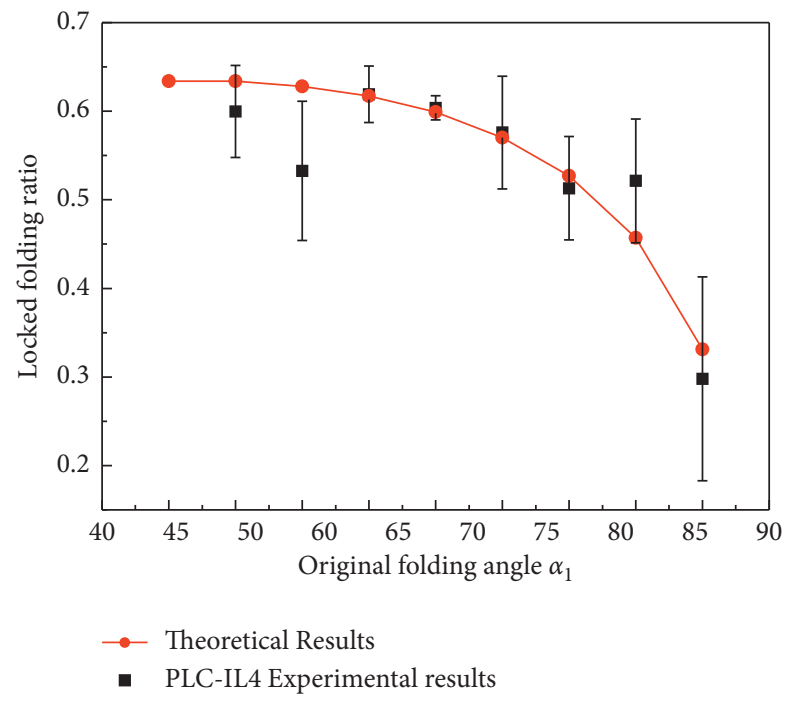

Figure 11: Comparison of the theoretical prediction locked folding ratio and experimental results.

predict the energy absorption of PLC-IL4 samples with different original folding angles.

Based on the deformation modes, the superfolding element theory [43], and the existing research [42, 44], the plastic energy dissipated by inclined plastic hinges, $E_{t}$, can be respectively calculated as follows:

$$
E_{t}=4 M_{P} \frac{(D / 4)^{2}}{r} \frac{1}{\tan \gamma_{1}^{0}} \int_{\beta_{1}^{0}}^{\pi / 2} \frac{\cos \beta_{1}}{\sin \alpha_{1}} \mathrm{~d} \beta_{1}+4 M_{P} \frac{(D / 4)^{2}}{r} \frac{1}{\tan \gamma_{2}^{0}} \int_{\beta_{2}^{0}}^{\pi / 2} \frac{\cos \beta_{2}}{\sin \alpha_{2}} \mathrm{~d} \beta_{2} .
$$



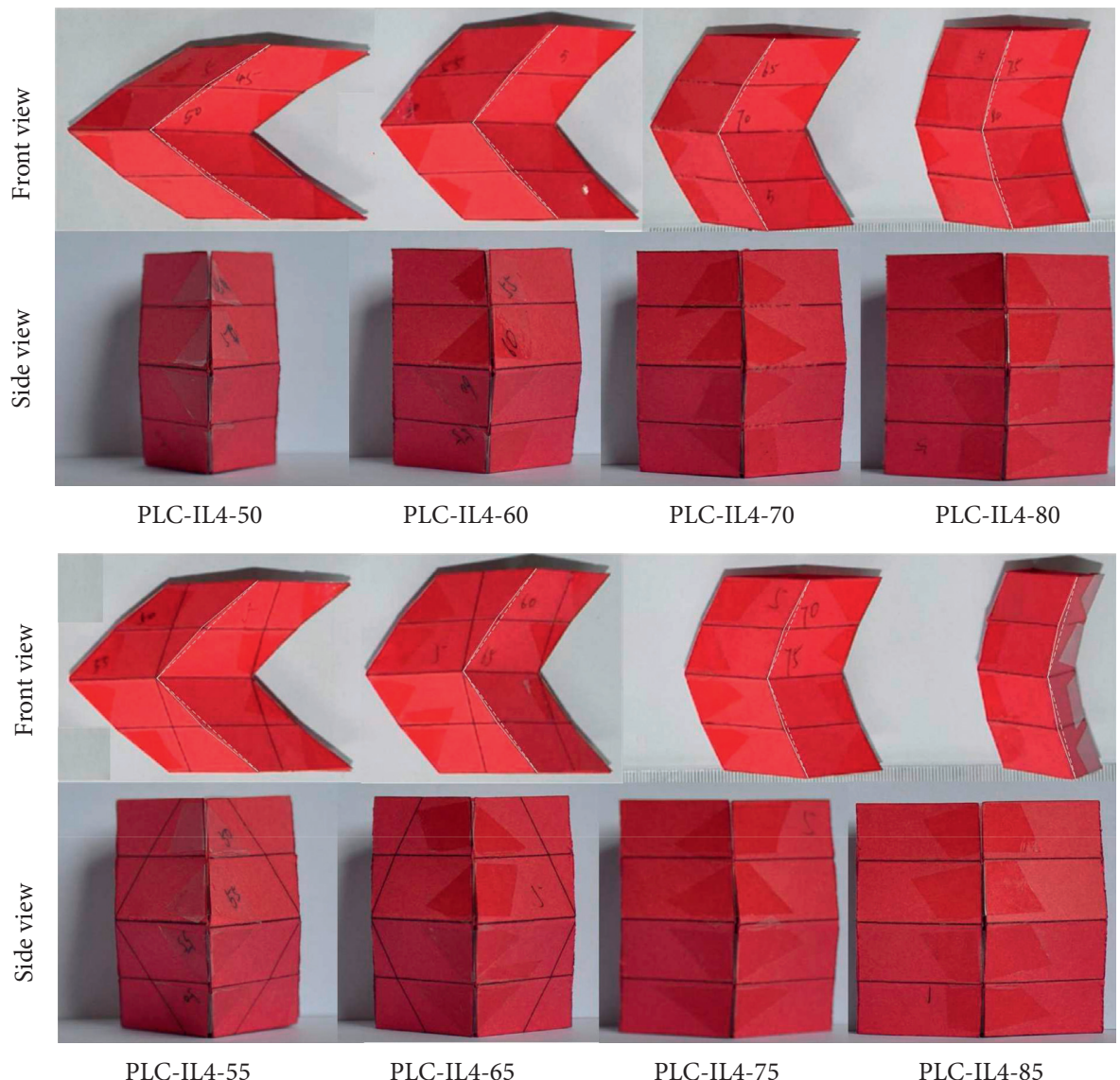

FIgURE 12: Typical deformation/failure modes of the PLC-IL4 origami structure with different original folding angles.

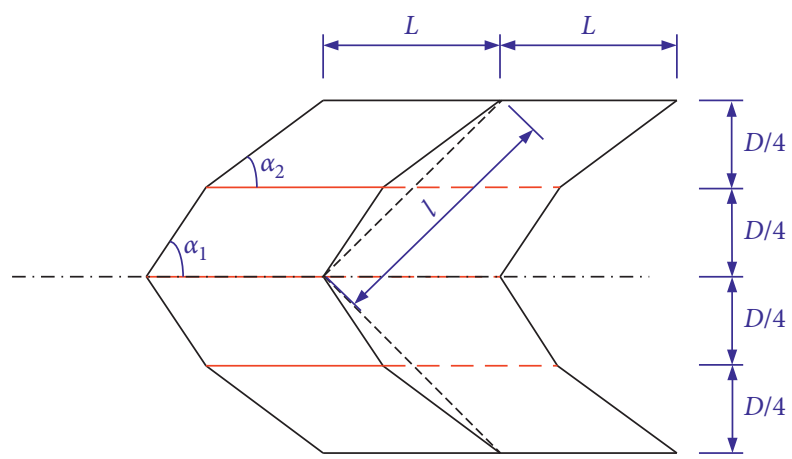

Figure 13: Typical deformation/failure modes of the PLC-IL4 origami unit cell.

TABLE 1: Comparison of theoretical prediction and experimental results of mean compressive force.

\begin{tabular}{lcccccc}
\hline Specimen type & $\delta 1(\mathrm{~mm})$ & $\delta 2(\mathrm{~mm})$ & $\mathrm{h} 0(\mathrm{~mm})$ & $E_{t}(\mathrm{~J})$ & $P_{m}^{t}(\mathrm{~N})$ & $P_{m}^{e}(\mathrm{~N})$ \\
\hline PLC-IL4-55-1 & 33.77 & 50 & 53.16 & 21.27 & 0.03742 & 3.158 \\
PLC-IL4-55-2 & 31.89 & 52.15 & 16.59 & 0.03742 & 2.410 \\
PLC-IL4-55-3 & 35.56 & 51.83 & 17.42 & 0.03742 & 3.090 \\
PLC-IL4-55-4 & 34.41 & 51.17 & 19.67 & 0.03742 & 2.943 \\
PLC-IL4-55-5 & 31.50 & 53.03 & 17.95 & 0.1016 & 2.606 \\
PLC-IL4-65-1 & 35.08 & 53.95 & 19.55 & 0.1016 & 7.754 \\
PLC-IL4-65-2 & 34.40 & 53.65 & 19.55 & 0.1016 & 7.119 \\
PLC-IL4-65-3 & 36.10 & 53.68 & 20.39 & 0.1016 & 7.119 \\
PLC-IL4-65-4 & 33.29 & & & 6.820 \\
\hline
\end{tabular}


TABle 1: Continued.

\begin{tabular}{lcccccc}
\hline Specimen type & $\delta 1(\mathrm{~mm})$ & $\delta 2(\mathrm{~mm})$ & $\mathrm{h} 0(\mathrm{~mm})$ & $E_{t}(\mathrm{~J})$ & $P_{m}^{t}(\mathrm{~N})$ & $P_{m}^{e}(\mathrm{~N})$ \\
\hline PLC-IL4-65-5 & 32.44 & 53.26 & 20.82 & 0.1016 & 6.685 \\
PLC-IL4-75-1 & 31.58 & 54.14 & 22.56 & 0.1767 & 10.729 \\
PLC-IL4-75-2 & 31.86 & 54.55 & 22.69 & 0.1767 & 10.668 \\
PLC-IL4-75-3 & 33.03 & 54.51 & 21.48 & 0.1767 & 11.269 \\
PLC-IL4-75-4 & 33.20 & 54.39 & 21.19 & 0.1767 & 11.423 \\
PLC-IL4-75-5 & 28.04 & 55.00 & 26.96 & 0.1767 & 8.978 \\
PLC-IL4-85-1 & 14.35 & 54.59 & 40.24 & 0.5611 & 1.798 \\
PLC-IL4-85-2 & 16.47 & 55 & 38.53 & 0.5611 & 2.191 \\
PLC-IL4-85-3 & 22.21 & 54.79 & 32.58 & 0.5611 & 19.949 \\
PLC-IL4-85-4 & 14.93 & 54.72 & 39.79 & 0.5611 & 23.592 \\
PLC-IL4-85-5 & 13.19 & 54.55 & 41.36 & 0.5611 & 19.317 \\
\hline
\end{tabular}

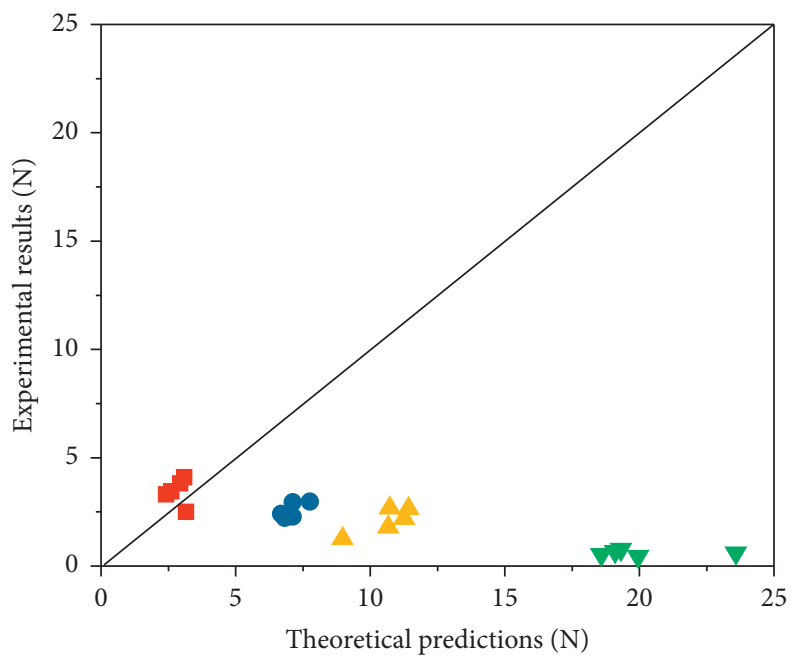
- PLC-IL4-55
A PLC-IL4-75
PLC-IL4-65
$\checkmark$ PLC-IL4-85

Figure 14: Comparison of the theoretical predictions and experimental results.
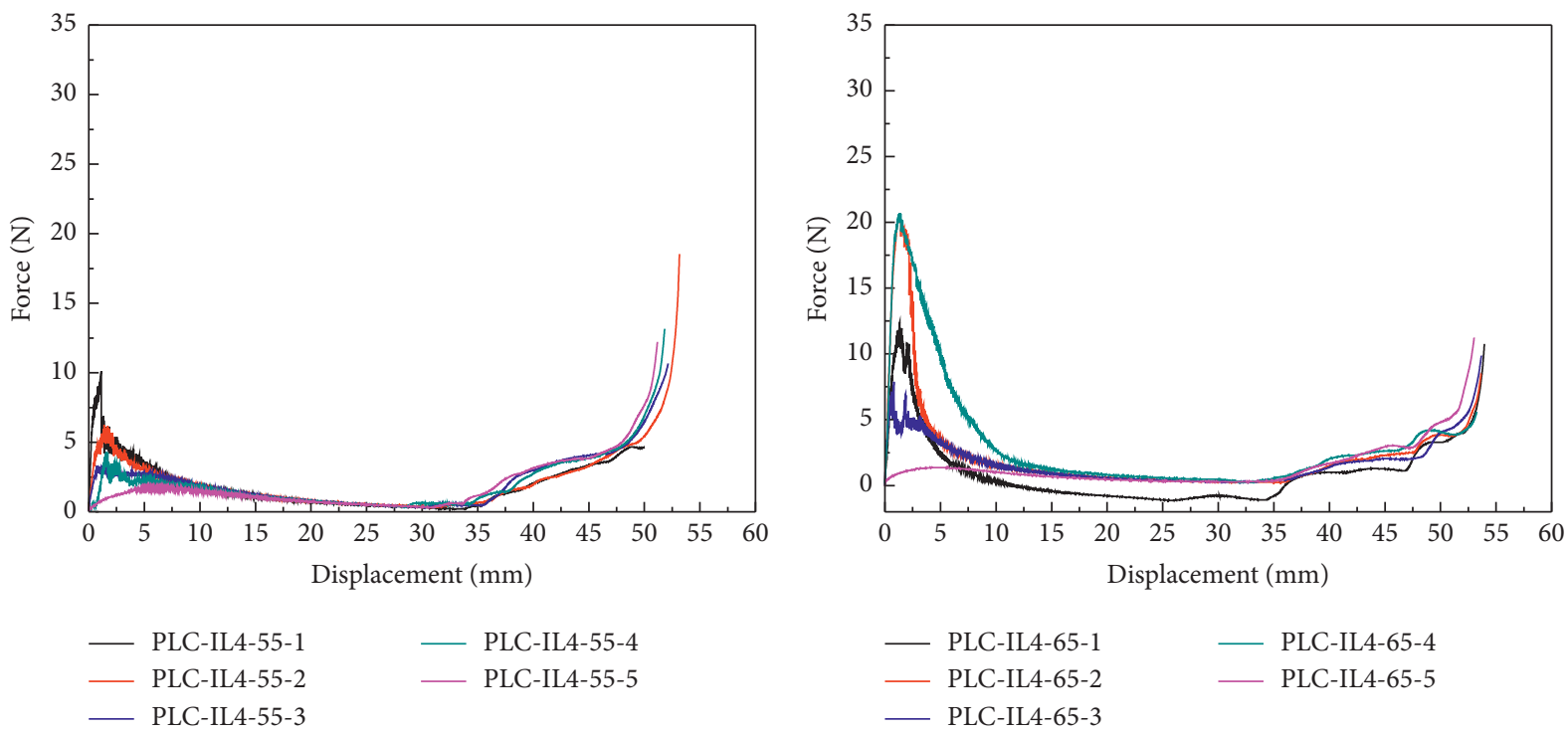

(b)

(a)

FIgURE 15: Continued. 


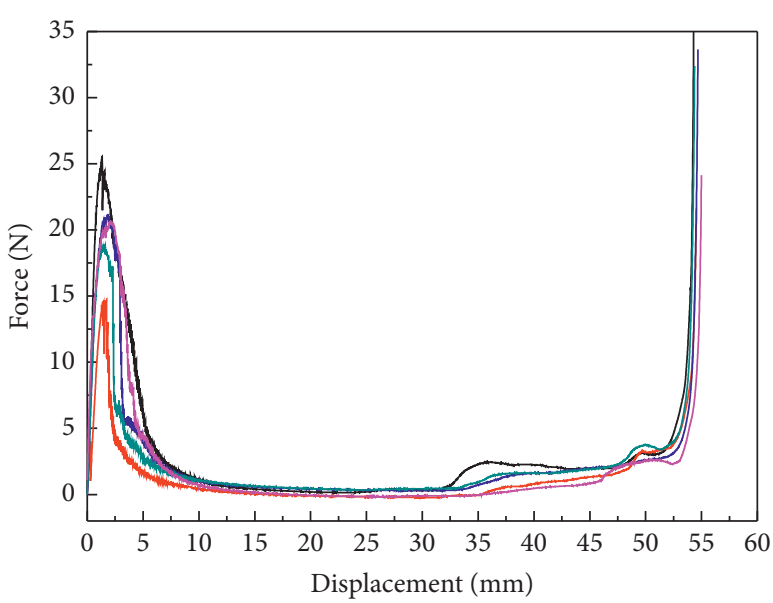

\begin{tabular}{l} 
— PLC-IL4-75-1 \\
P PLC-IL4-75-2 \\
\hline PLC-IL4-75-3
\end{tabular}

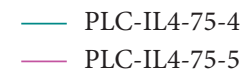

(c)

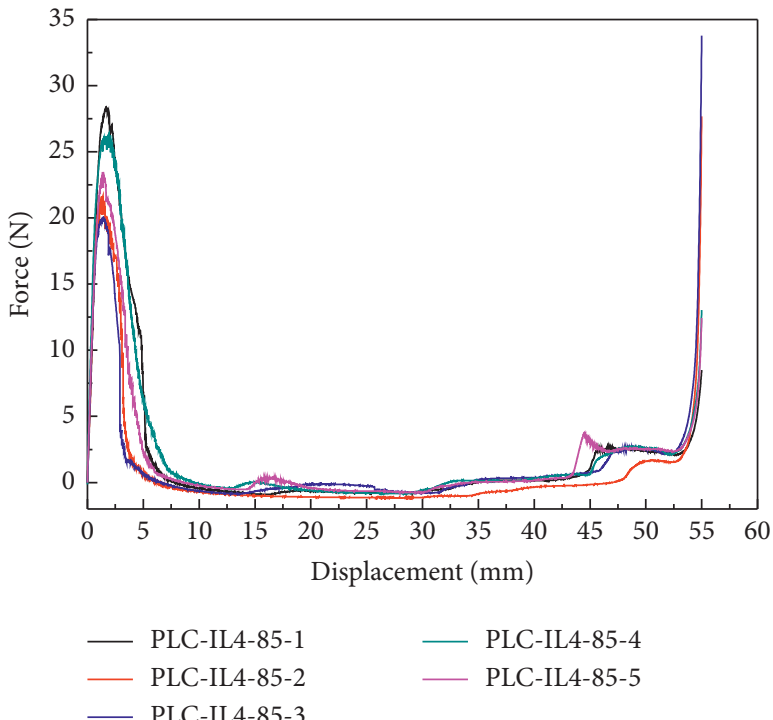

(d)

FIgURE 15: Quasi-static compressive curves of samples with different original folding angles.

The energy balance between external work done by the compressive force and energy absorption in PLC-IL4 samples is applied, and the mean compressive force can be obtained by dividing the total energy absorption by the final compression displacement.

$$
P_{m}^{t} \cdot 0.73 h_{0}=E_{t} .
$$

The experimental quasi-static mean compressive force can be calculated as follows:

$$
P_{m}^{e}=\frac{\int_{\delta_{1}}^{\delta_{2}} P(x)}{\delta_{2}-\delta_{1}},
$$

where $\delta_{1}$ and $\delta_{2}$ are starting point and the destination of the plastic deformation process, and $h_{0}=\delta_{2}-\delta_{1}$.

The theoretical predictions, $P_{m}^{t}$, calculated from equation (16) and the experimental results, $P_{m}^{e}$, obtained from equation (17), of samples are listed in Table 1.

The prediction mean compression forces are compared with the experimental results, as shown in Figure 14. It can be seen that the data points of PLC-IL4-55 specimens are concentrated basically around a straight line with a slope of 1 , herein indicating a reasonable correlation between the experimental compression force and the predicted value of mean compression forces. However, for the large angle case, there is a greater difference between the theoretical predictions and the experimental results. The difference between the theoretical predictions and the experimental results becomes larger with the increasing original folding angle. It is evident that the theoretical prediction of the large angle specimens tends to overestimate the mean compression force. The main reason is as follows: the first reason is that the effect of friction is not considered throughout the folding process, and it is impossible to overcome friction in the experiment. The second reason is the large original folding angle specimens undergo a large plastic deformation in the initial folding process, which is not taken into account in the approximate theoretical model of the elastic-plastic deformation stage, as shown in Figures 15 and 16. The third is the deformation pattern gradually changes from bending to buckling with the increasing original folding angle. For this reason, the theoretical model in this study is only suitable for designing origami structures with small angles $\left(<60^{\circ}\right)$, and structures with large angles require modification of the theoretical model due to the transformation of the deformation mode. The corresponding theoretical consideration, as a part of our current and future work, is worthy to be further conducted. 
PLC-IL4-55

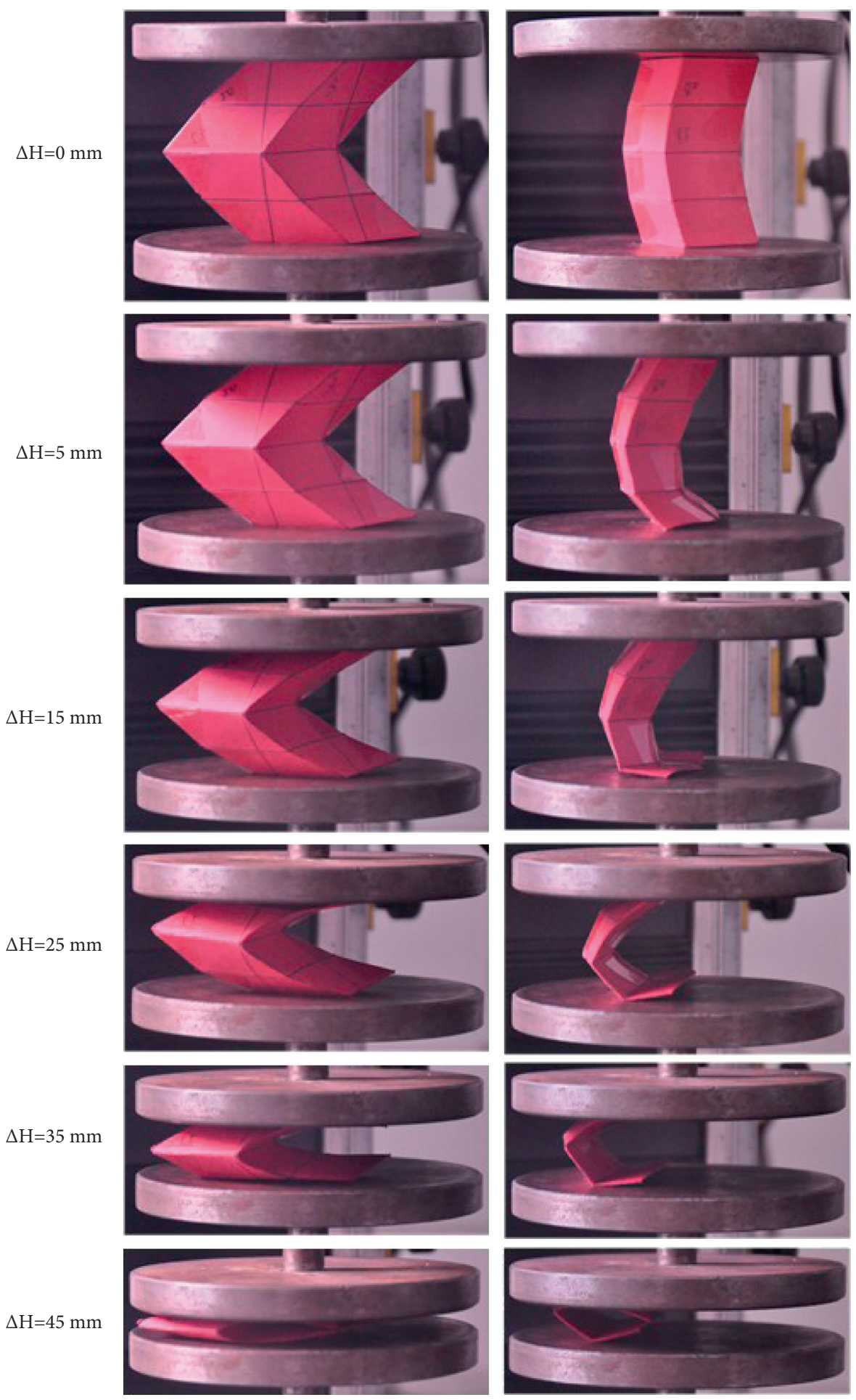

Figure 16: Deformation process comparison of PLC-IL4-55 and PLC-IL4-85. 


\section{Conclusion}

The quasi-static mechanical behavior of polyline crease origami self-locking structures with different original folding angles and length-to-height ratios under axial compression loading was investigated. The out-plane Poisson's ratio $v_{\mathrm{ZX}}$ of PLC-IL4 origami structures exhibits a negative Poisson ratio under axial compression deformations, while the $v_{\mathrm{ZY}}$ exhibits a positive Poisson ratio. It is obtained that the original folding angle has a great impact on Poisson's ratio than that of the length-to-height ratio. The PLC-IL4 origami structures can design on request self-locking behavior and original folding angles, which can be exploited for the purpose of intelligent control and metamaterial design.

The different load capacity and failure modes of the PLCIL4 with different original folding angles are discussed through the experimental results. It is found that the load capacity of samples is mainly controlled by the original folding angle values, while the deformation/failure modes are very similar to each other. Based on the experiments, an approximate mathematical formula was established to predict the mean compression force for every type of sample. A reasonable agreement between the theoretical predictions and experimental results is obtained.

\section{Data Availability}

The data used to support the findings of this study are available from the corresponding author upon request.

\section{Conflicts of Interest}

The authors declare that they have no conflicts of interest.

\section{Acknowledgments}

The authors thank Dr. Zhixun Yang in Harbin Engineering University, for many helpful discussions. This work was supported by the Scientific and Technological Innovation Programs of Higher Education Institutions in Shanxi (Grant No. 2019L0497) and the Youth Foundation of Shanxi University of Finance and Economics (QN-2019005). The financial contributions are gratefully acknowledged.

\section{References}

[1] J. T. B. Overvelde, J. C. Weaver, C. Hoberman, and K. Bertoldi, "Rational design of reconfigurable prismatic architected materials," Nature, vol. 541, no. 7637, pp. 347-352, 2017.

[2] E. T. Filipov, T. Tachi, and G. H. Paulino, "Origami tubes assembled into stiff, yet reconfigurable structures and metamaterials," Proceedings of the National Academy of Sciences, vol. 112, no. 40, pp. 12321-12326, 2015.

[3] S. Waitukaitis, R. Menaut, B. G. Chen, and M van Hecke, "Origami multistability: from single vertices to metasheets," Physical Review Letters, vol. 114, Article ID 055503, 2015.

[4] E. Boatti, N. Vasios, and K. Bertoldi, "Origami metamaterials for tunable thermal expansion," Advanced Materials, vol. 29, no. 26, Article ID 1700360, 2017.
[5] S. Li, H. Fang, and K. W. Wang, "Recoverable and programmable collapse from folding pressurized origami cellular solids," Physical Review Letters, vol. 117, no. 11, Article ID 114301, 2016.

[6] J. L. Silverberg, A. A. Evans, L. McLeod et al., "Using origami design principles to fold reprogrammable mechanical metamaterials," Science, vol. 345, no. 6197, pp. 647-650, 2014.

[7] L. H. Dudte, E. Vouga, T. Tachi, and L. Mahadevan, "Programming curvature using origami tessellations," Nature Materials, vol. 15, no. 5, pp. 583-588, 2016.

[8] J. M. Gattas, W. Wu, and Z. You, "Miura-base rigid origami: parameterizations of first level derivative and piecewise geometries," Journal of Mechanical Design, vol. 135, Article ID 111011, 2013.

[9] J. M. Gattas and Z. You, "The behaviour of curved-crease foldcores under low-velocity impact loads," International Journal of Solids and Structures, vol. 53, no. 15, pp. 80-91, 2015.

[10] Z. Y. Wei, Z. V. Guo, L. Dudte, H. Y. Liang, and L. Mahadevan, "Geometric mechanics of periodic pleated origami," Physical Review Letters, vol. 110, no. 21, Article ID 215501, 2013.

[11] M. Schenk and S. D. Guest, "Geometry of miura-folded metamaterials," Proceedings of the National Academy of Sciences, vol. 110, no. 9, pp. 3276-3281, 2013.

[12] C. Lv, D. Krishnaraju, G. Konjevod, H. Yu, and H. Jiang, "Origami based mechanical metamaterials," Scientific Reports, vol. 4, no. 1, p. 5979, 2014.

[13] M. Eidini and G. H. Paulino, "Unraveling metamaterial properties in zigzag-base folded sheets," Science Advances, vol. 1, no. 8, p. 1500224, 2015.

[14] B. G.-g. Chen, B. Liu, A. A. Evans et al., "Topological mechanics of origami and kirigami," Physical Review Letters, vol. 116, no. 13, Article ID 135501, 2016.

[15] Z. Y. Wei, Z. V. Guo, L. Dudte, H. Y. Liang, and L Mahadevan, "Geometric mechanics of periodic pleated origami," Physical Review Letters, vol. 110, p. 215501, 2013.

[16] H. Fang, S. A. Chu, Y. Xia, and K. W Wang, "Programmable self-locking origami mechanical metamaterials," Advanced Materials, vol. 30, Article ID e1706311, 2018.

[17] H. Fang, S. Li, and K. W. Wang, "Self-locking degree-4 vertex origami structures," Proceedings of the Royal Society A: Mathematical, Physical \& Engineering Sciences, vol. 472, no. 2195, Article ID 20160682, 2016.

[18] J. Kim, D. Y. Lee, S. R. Kim, and K. Cho, "A self-deployable origami structure with locking mechanism induced by buckling effect," Proceedings of the IEEE, vol. 58, pp. 31663171, 2015.

[19] H. Yasuda and J. Yang, "Reentrant origami-based metamaterials with negative Poisson's ratio and bistability," Physical Review Letters, vol. 114, no. 18, Article ID 185502, 2015.

[20] Y. Tang and J. Yin, "Design of cut unit geometry in hierarchical kirigami-based auxetic metamaterials for high stretchability and compressibility," Extreme Mechanics Letters, vol. 12, pp. 77-85, 2017.

[21] L. Mizzia, E. Salvatib, A. Spaggiari, C. Tan, and M. Korsunsky, "Highly stretchable two-dimensional auxetic metamaterial sheets fabricated via direct-laser cutting," International Journal of Mechanical Sciences, vol. 167.

[22] S. Waitukaitis and M. van Hecke, "Origami building blocks: generic and special four-vertices," Physical Review A, vol. 93, no. 2, Article ID 23003, 2016. 
[23] K. E. Evans and A. Alderson, "Auxetic materials: functional materials and structures from lateral thinking!" Advanced Materials, vol. 12, no. 9, pp. 617-628, 2000.

[24] A. Lorato, P. Innocenti, F. Scarpa et al., "The transverse elastic properties of chiral honeycombs," Composites Science and Technology, vol. 70, no. 7, pp. 1057-1063, 2010.

[25] K. W. Wojciechowski, "Two-dimensional isotropic system with a negative Poisson ratio," Physics Letters A, vol. 137, no. 1-2, pp. 60-64, 1989.

[26] P. P. Pratapa, K. Liu, and G. H. Paulino, "Geometric mechanics of origami patterns exhibiting Poisson's ratio switch by breaking mountain and valley assignment," Physical Review Letters, vol. 122, no. 15, Article ID 155501, 2019.

[27] J. Zhang, G. Lu, Y. Zhang, and Z. You, "A study on ballistic performance of origami sandwich panels," International Journal of Impact Engineering, vol. 156, Article ID 103925, 2021.

[28] J. Zhang, D. Karagiozova, G. Lu, and P. Chen, "Quasi-static in-plane compression of zig-zag folded metamaterials at large plastic strains," Thin-Walled Structures, vol. 159, no. 2, Article ID 107285, 2020.

[29] J. Zhang, G. Lu, and Z. You, "Large deformation and energy absorption of additively manufactured auxetic materials and structures: a review," Composites Part B: Engineering, vol. 201, no. 4, Article ID 108340, 2020.

[30] J. Zhang and G. Lu, "Dynamic tensile behaviour of re-entrant honeycombs," International Journal of Impact Engineering, vol. 139, Article ID 103497, 2020.

[31] L. Jing, X. Su, D. Chen, F. Yang, and L. Zhao, "Experimental and numerical study of sandwich beams with layered-gradient foam cores under low-velocity impact," Thin-Walled Structures, vol. 135, pp. 227-244, 2019.

[32] L. Jing, X. Su, F. Yang, H. Ma, and L. Zhao, "Compressive strain rate dependence and constitutive modeling of closedcell aluminum foams with various relative densities," Journal of Materials Science, vol. 53, no. 20, pp. 14739-14757, 2018.

[33] L. Jing, Z. Wang, and L. Zhao, "The dynamic response of sandwich panels with cellular metal cores to localized impulsive loading," Composites Part B: Engineering, vol. 94, pp. 52-63, 2016.

[34] P. Zhang, Z. Wang, and L. Zhao, "Dynamic crushing behavior of open-cell aluminum foam with negative Poisson's ratio," Applied Physics A, vol. 123, no. 5, p. 321, 2017.

[35] P. Zhang, X. Li, T. Jin, Z. Wang, and L. Zhao, "Dynamic response of circular metallic sandwich panels under projectile impact," Journal of Sandwich Structures and Materials, vol. 19, no. 5, pp. 572-594, 2017.

[36] X. Li, P. Zhang, L. Shiqiang, Z. Wang, and G. Wu, "Dynamic response of aluminum honeycomb sandwich panels under foam projectile impact," Mechanics of Advanced Materials and Structures, vol. 25, no. 8, pp. 637-646, 2018.

[37] D. Karagiozova and M. Alves, "On the dynamic compression of cellular materials with local structural softening," International Journal of Impact Engineering, vol. 108, pp. 153-170, 2017.

[38] M. Schenk, S. D. Guest, and G. J. McShane, "Novel stacked folded cores for blast-resistant sandwich beams," International Journal of Solids and Structures, vol. 51, no. 25-26, pp. 4196-4214, 2014

[39] P. Zhang, X. Li, Z. Wang, L. Zhao, and X. Yan, "Dynamic blast loading response of sandwich beam with origami-inspired core," Results in Physics, vol. 10, pp. 946-955, 2018.

[40] J. Zhang, D. Karagiozova, Z. You, Y. Chen, and G. Lu, "Quasistatic large deformation compressive behaviour of origami- based metamaterials," International Journal of Mechanical Sciences, vol. 153-154, pp. 194-207, 2019.

[41] D. Karagiozova, J. Zhang, G. Lu, and Z. You, "Dynamic inplane compression of miura-ori patterned metamaterials," International Journal of Impact Engineering, vol. 129, pp. 80-100, 2019.

[42] G. Lu and T. X. Yu, Energy Absorption of Structures and Materials, Woodhead Publishing, Cambridge, UK, 2003.

[43] T. Wierzbicki and W. Abramowicz, "On the crushing mechanics of thin-walled structures," Journal of Applied Mechanics, vol. 50, pp. 727-734, 1983.

[44] J. Ma, D. Hou, Y. Chen, and Z. You, "Quasi-static axial crushing of thin-walled tubes with a kite-shape rigid origami pattern: numerical simulation," Thin-Walled Structures, vol. 100, pp. 38-47, 2016. 